\title{
Descriptive Analysis of Skin Temperature Variability of Sympathetic Nervous System Activity in Stress
}

\author{
Karthikeyan Palanisamy, ME ${ }^{1)}$, Murugappan Murugappan, $\mathrm{PhD}^{1)}$, Sazali Yaacob, PhD ${ }^{1)}$ \\ 1) School of Mechatronics Engineering, Universiti Malaysia Perlis: Ulu pauh, Kangar, Perlis 02600, \\ Malaysia.E-mail: karthi_209170@yahoo.com
}

\begin{abstract}
Purpose] Stress is a common factor of several diseases. Stress can be reduced through appropriate stress management and relaxation methods. In this study, variation in skin temperature (ST) was investigated as a primary measure for identifying changes in stress levels. Our results should be helpful for the development of a stress measurement tool based on multimodal signals. [Subjects] Sixty healthy volunteers (30 females and 30 males) of three different races (Malay, Chinese, and Indian) with a mean age of $22.5 \pm 2.5$ years participated in this study. [Methods] The Stroop color word test was used to design a data acquisition protocol of 12.36 min for this experiment. ST variation was measured continuously during the Stroop colour word test and statistical features were computed. Further, descriptive analysis and stress levels were classified using a Probabilistic Neural Network (PNN) to find the optimum features. [Results] Among the 60 subjects, the mean ST of 48 subjects (80\%) rose linearly from the normal state to the high-stress state. In addition, Malay subjects were more sensitive to stress than other two races as measured by the mean skin temperature. A maximum mean classification rate of $88 \%$ was achieved for the four different stress levels on all the subjects using PNN. [Conclusion] Our investigation proves that the mean ST is a reliable measure for identifying stress level changes and may be useful for designing a multimodal stress measurement system.
\end{abstract}

Key words: Human stress level, Skin temperature, Stroop colour word test

(This article was submitted Jul. 31, 2012, and was accepted Aug. 30, 2012)

\section{INTRODUCTION}

Skin conductance in human is not homogenous, and skin temperature is not uniform throughout the human body ${ }^{1)}$ varying by $2{ }^{\circ} \mathrm{C}$ to $4{ }^{\circ} \mathrm{C}$ at different surfaces of the skin $^{2)}$. In general, human body activation and mobilization are controlled by the sympathetic nervous system (SNS), whereas relaxation and restoration are controlled by the parasympathetic nervous system (PNS). Various activities of both SNS and PNS are reflected in the autonomic nervous system (ANS) such as stress, and emotion ${ }^{3)}$. Changes in physiological states due to stress are mainly reflected in ANS activities. Several studies have revealed the clinical and physiological relationships between stress and ANS activity ${ }^{4,5)}$. Recently, identifying and measuring the level of stress through physiological signals has become an emerging field in psychophysiology. Physiological signals such as galvanic skin response (GSR) and skin temperature (ST) are considered to be reliable indicators of stress in addition to ECG, respiration rate (RR), electromyogram (EMG), and blood pressure (BP) signals ${ }^{6,7)}$. Heart rate variability (HRV) and BP have been reported to be prime predictors of the stress by several studies ${ }^{4-8}$. BP is often measured at certain intervals during stress inducing tasks ${ }^{9,10)}$. ST is a simple signal of stress compared to other physiological signals. Measurement of BP is limited by simultaneous data acquisition and RR by obtrusive electrode placement location. The characteristics of ST are affected by factors such as environment, internal core temperature, muscle tone, and evaporation ${ }^{2}$. However, the identification of the core temperature of the body by measuring the skin surface is advantageous for non-invasive measurement of several affective states such as, stress, emotion and anxiety ${ }^{11)}$. A few studies have reported a relationship between stress and ST but without comparison with HRV, GSR or photoplethysmography (PPG) ${ }^{2-6)}$.

The Stroop colour word test is a stress-inducing method which is widely used in human stress assessment studies ${ }^{6-12)}$. Different colours of the word displayed on colour boxes and the corresponding pronunciation on the particular color plays important role increases mental demands which induces the stress in the subjects. Zhai et al. used three physiological signals (ST, HRV, and GSR) to identify stress in Stroop color and word test using support vector machine (SVM), naive Bayes and decision tress classifiers. They obtained classification accuracy of $90.02 \%$ in all signals without have discussion with individual signal efficiency for detect the stress $^{6)}$.

In many of the above studies, ST was used as a secondary measure in various researches and its relationship with stress and other stress indicators was not discussed ${ }^{2-6)}$. However, due to its simple characteristics, it can be used to develop a 
Table 1. Previous studies of skin temperature in stress assessment

\begin{tabular}{cllll}
\hline Ref. & Subject and protocol details & Physiological signals & ST features used & Significance \\
\hline 10) & $\begin{array}{l}\text { 10 subjects and limited-time } \\
\text { serial subtraction task }\end{array}$ & ST and PPG & Mean ST & ST is a simple measure of stress \\
\hline 5) & $\begin{array}{l}\text { 32 subjects, Stroop colour word } \\
\text { test }\end{array}$ & GSR, BVP, and ST & Mean ST & Overall ST was elevated during stress \\
\hline 6$)$ & 70 subjects, Coin stacking task & ECG, EMG, GSR, and ST & - & $\begin{array}{l}\text { Increased peripheral skin temperature } \\
\text { in the high stress state }\end{array}$ \\
\hline
\end{tabular}

biofeedback control system to control autonomic activity in therapy procedures. In the present study, we attempted to resolve the limitations described above, by investigating (i) subject to subject variation of all the participating subjects, (ii) overall variation among the three races, and (iii) classification of stress levels using optimal features in the 60 subjects.

\section{SUBJECTS AND METHODS}

Changes in ANS activities through physiological signals have mostly been investigated by assessing the changes in affective state (emotion and stress) ${ }^{4-14)}$. The correlation between changes in affective state and ANS activities can be easily estimated through two different approaches, 'subject dependent' (post-task questionnaire) and 'subject independent' (protocol based). ST is a simple and effective way of identifying stress changes through a non-invasive procedure. Indeed, filtering and processing of ST signal is also easier compared than the processes used for other physiological signals. Hence, we focused on analyzing ST variations with stress changes in this study, rather than compared to other physiological signals.

ST responds linearly to the contraction and relaxation of the smooth muscles surrounding the blood vessels. The skin temperature is either increases or decreases based in direct relationship with blood flow in blood vessels. When the blood flow increases the temperature increases and vice versa. Environmental conditions, evaporation, muscle tone, and core temperature are possible confounding factors in skin temperature assessment of affective states including stress. In stress research, the surface skin temperature measurement of internal core temperature is measured because it is related to the effect of stress on the adrenal hypo thalamus (ATH) axis. This ATH activation is a prime way through which SNS activity influences ANS under stress. Changes in continuous skin temperature measurement may not perfectly reflect peripheral vasomotor at all times and this will reduce the sensitivity of the ST-based stress level detection. Table 1 displays the previous studies which have investigated skin temperature in stress research and their conclusions.

In this study, stress was induced using the Stroop color word test. The stress levels of the subjects were increased by increasing mental demand. The experimental protocol consisted of four different levels; a relaxation (normal) state with soft music, and low, medium, and high levels of stress. The time of each block appeared on the screen was gradually reduced according to the level, for the subject to orally describe the color of the box but not the word or world color. The time for the low stress level was $128 \mathrm{sec}$, for the medium stress level was $64 \mathrm{sec}$, and for the high stress level was 32 seconds. These differences in time are important for increasing the mental load in vocalizing the correct color in time ${ }^{6-12)}$. Each level has two sessions consisting of congruent Stroop and incongruent Stroop. The experimental protocol was reviewed by our university ethical committee.

In this work, we included the subjects from three different races namely Malay, Chinese, and Indian. Sixty healthy volunteers ( 30 females and 30 males) with 20 subjects from each race participated in this study. All the subjects were in the age range of 20-25 years; they were not taking any medication, and had no drug or smoking habits. All were free of symptoms, signs or history of cardiovascular and mental diseases. A set of questions was asked of the subjects to identify their mental and physical condition before starting the experiment. All the subjects were given a brief explanation about this research and provided their consent to for participation in this experiment. The study protocol followed local ethical regulations. Initially, the subjects sat comfortably on a chair in front of a LCD screen projector. Before placing electrodes on subjects' skin, all the subjects were requested to rest for of $30 \mathrm{~min}$, and the room temperature was maintained constantly at $24{ }^{\circ} \mathrm{C}$ throughout the experiment. ECG, EMG, GSR and ST of all of the participants were measured. In this study, we focused on analyzing the ST characteristics for stress assessment. In our previous study, the effects of ECG and EMG were studied using the same protocol ${ }^{12-15)}$. A theremistor based temperature sensor was placed under the armpit of each subject to avoid external temperature influences. The temperature data was sampled at $1 \mathrm{kHz}$ using the device of AD Instruments, Australia.

In previous studies, researchers have analyzed ST signals at a maximum frequency of $0.1 \mathrm{~Hz}$ estimate the stress level changes ${ }^{2)}$. An elliptic band-pass filter was used to preprocess the ST signal in the frequency range from $0.005-0.1 \mathrm{~Hz}$. As a preliminary study, four statistical features such as mean, standard deviation, minimum and maximum of ST were investigated.

PNN is a simple classifier based on the probability density function. It has input, pattern, summation and output units. All these units are interconnected and the pattern unit is activated based on the computed distance from the input vector to the testing vectors. PNN works based on smoothing parameter or spread factor, this factor can be used to decide 
Table 2. Results of PNN of stress level classification using mean skin temperature

\begin{tabular}{|c|c|c|c|c|c|c|}
\hline \multirow[t]{2}{*}{ Spread factor } & \multirow[t]{2}{*}{ Features } & \multirow[t]{2}{*}{ Normal (\%) } & \multicolumn{3}{|c|}{ Stress Levels } & \multirow{2}{*}{$\begin{array}{l}\text { Overall } \\
\text { accuracy }\end{array}$} \\
\hline & & & Low $(\%)$ & Medium (\%) & High $(\%)$ & \\
\hline \multirow{4}{*}{0.05} & Mean & 88.33 & 55.00 & 11.67 & 93.33 & 62.08 \\
\hline & Maximum & 88.33 & 53.33 & 6.67 & 93.33 & 60.42 \\
\hline & Minimum & 90.00 & 51.67 & 11.67 & 80.00 & 58.33 \\
\hline & Standard Deviation & 16.67 & 5.00 & 5.00 & 66.67 & 23.33 \\
\hline \multirow{4}{*}{0.01} & Mean & 93.33 & 73.33 & 46.67 & 80.00 & 73.33 \\
\hline & Maximum & 96.67 & 71.67 & 50.00 & 81.67 & 75.00 \\
\hline & Minimum & 95.00 & 78.33 & 35.00 & 80.00 & 72.08 \\
\hline & Standard Deviation & 46.67 & 6.67 & 6.67 & 96.67 & 39.17 \\
\hline \multirow{4}{*}{0.005} & Mean & 96.67 & 76.67 & 58.33 & 71.67 & 75.83 \\
\hline & Maximum & 98.33 & 71.67 & 56.67 & 81.67 & 77.08 \\
\hline & Minimum & 96.67 & 78.33 & 55.00 & 75.00 & 76.25 \\
\hline & Standard Deviation & 56.67 & 15.00 & 15.00 & 63.33 & 37.50 \\
\hline \multirow{4}{*}{0.001} & Mean & 100.00 & 86.67 & 81.67 & 86.67 & 88.75 \\
\hline & Maximum & 98.33 & 83.33 & 71.67 & 91.67 & 86.25 \\
\hline & Minimum & 100.00 & 86.67 & 75.00 & 81.67 & 85.83 \\
\hline & Standard Deviation & 70.00 & 35.00 & 35.00 & 68.33 & 52.08 \\
\hline
\end{tabular}

the nonlinear decision boundaries of each classification problems. The complete architecture, and methodologies were developed by Specht ${ }^{16)}$.

\section{RESULTS}

The normalized mean temperature value used in for the statistical analysis to investigate the change in the transition from the normal state to the high stress state. Student's t-test was used to analyze the statistical changes between the stress levels. The statistical values for the change between normal to low stress were: degree of freedom $(\mathrm{DOF})=59$, $\mathrm{p}<0.0001, \mathrm{t}=-4.361$, Pearson correlation $=0.735$; between low to medium $\mathrm{DOF}=59, \mathrm{p}<0.0001, \mathrm{t}=-6.186$, Pearson correlation $=0.6733$ and between medium to high $\mathrm{DOF}=59$, $\mathrm{p}<.0001, \mathrm{t}=-5.087$, Pearson correlation $=0.575$. All the differences were statically significant $(p<0.0001)$. Furthermore, the Pearson correlation gradually decreased from normal state to the high stress state. The overall response to four classes was found $\mathrm{F}=30.80, \mathrm{DOF}=3 / 59, \mathrm{p}<0.0001$ by using the two-way ANOVA.

We used a simple PNN to classify the four different states of stress including one relaxation (normal). Table 2 shows the classification results of skin temperature variation with stress. The four features of all the stress levels of data were given to the input PNN for testing and training. The spread factor plays an important role in improving the accuracy of classifier. In this study, the spread factor value ranged from 0.05 to 0.001 . The overall accuracy was $62.08 \%$ whilst the spread factor is 0.05 . The overall resultant classification accuracy is improved up to $88.75 \%$ from $62.08 \%$ after reducing the spread factor from 0.05 to 0.001 as shown in Table 2 .

\section{DISCUSSION}

Analysis of variation between subjects performance in experiments is a major issue in computational biology. In general, human functionalities differ due to several factors including lifestyle, living environment, food, age, gender. Most of affective states (stress, emotion, drowsiness) research relies on subjective responses and it is hard to define a common ground truth in this research. However, in this in the present study, we incorporated the subjectindependent analysis. A common stress inducement stimulus was chosen for all the 60 subjects who were from identical living backgrounds in three different races (Malay, Chinese, and Indian). The results of subjective analysis show that $80 \%$ (48) of subjects' mean skin temperature gradually increased in the transition from the normal state to the high stressful state. Six subjects mean skin temperature levels responded inversely to the stimuli and 6 subjects had oscillatory or no changes in response to the stimuli. We attribute the anomalous variations of ST in subjects to data fluctuations that may have arisen from subjects' backgrounds and subjects' psychophysiological states. Specifically, the mean ST of one Malay subject, three Chinese subjects and eight Indian subjects did not to the stress stimuli at the four different levels (normal, and low, medium, and high stress). The mean skin temperature of Malay, Chinese, and Indian subjects increased gradually in $95 \%, 85 \%$ and $60 \%$ respectively. This result indicates that, the Malay subjects were more stressed by the Stoop colour task than subjects of the other two races.

The mean, maximum, minimum skin temperature gave improved classification rates of $88.75 \%, 86.25 \%$, and $85.83 \%$ respectively according to the PNN classifier. The accuracy of the normal state was almost $100 \%$ and, the overall performance of mean skin temperature feature was 
better for all the four stress states than those of the other features. Individual classification results of normal, low, medium, and high stress states were 100\% 86.67\%, 81.67\%, and $86.67 \%$ respectively, when the spread value of the PNN classifier was 0.001 .

This study performed a detailed statistical analysis of skin temperature to distinguish the different stress levels of 60 subjects. Four ST features were investigated to assess the stress level changes of the subjects. The mean skin temperature gradually increased in most subjects from as stress transitioned from a normal state to a high stress state while subjects performed the Stroop color word test. In the comparison of the three races, the Indian subjects were highly subjective by measuring the mean skin temperature changes with stress levels and this may be because of a skin property of this race. The simple PNN was used to classify the features of skin temperature and the result showed an overall classification accuracy of $88.75 \%$ for mean ST. Therefore, mean skin temperature can be considered as to be indicative of for stress and it could be used as a simple variable to identify stress level changes in SNS activity. Combining other physiological signals with mean skin temperature may lead to the development of a more efficient stress level measurement system.

\section{REFERENCES}

1) Sessler DI: Temperature monitoring and perioperative thermoregulation Anesthesiology, 2008, 109: 318-338. [Medline] [CrossRef]

2) Shusterman V, Barnea O: Sympathetic nervous system activity in stress and biofeedback relaxation. IEEE Eng Med Biol Mag, 2005.

3) Alvarsson JJ, Wiens S, Nilsson ME: Stress recovery during exposure to nature sound and environmental noises. Int J Environ Res Public Health, 2010, 7: 1036-1046. [Medline] [CrossRef]

4) Healey JA, Picard RW: Detecting stress during real-world driving tasks using physiological sensors. IEEE Trans Intell Transp Syst, 2005, 6:
156-166. [CrossRef]

5) Lundberg U, Melin B: Psychophysiological stress and emg activity of the trapezius muscle. Int J Behav Med, 1994, 1: 354-370. [Medline] [CrossRef]

6) Zhai J, Barreto A: Stress detection in computer users based on digital signal processing of noninvasive physiological variables, in engineering in medicine and biology society, 2006. EMBS '06. 28th Annual International Conference of the IEEE, 2006, 1355-1358.

7) Sul A, Shin J, Lee C, et al.: Evaluation of stress reactivity and recovery using biosignals and fuzzy theory, in 24th Annual Conference and the Annual Fall Meeting of the Biomedical Engineering Society EMBS/BMES Conference, 2002 Proceedings of the Second Joint Engineering in Medicine and Biology, 2002, 32-33.

8) Seong HM, Lee JS, Shin TM, et al: The analysis of mental stress using time-frequency distribution of heart rate variability signal, in proceedings of the 26th annual international conference of the IEEE EMBS, San Francisco, CA, USA September 1-5, 2004, 283-284.

9) Tulen HM, Moleman P, Steenist HGV, et al.: Characterization of Stress Reactions to the Stroop Color Word Test. Pharmacol Biochem Behav, 1989, 32: 9-15.

10) Pehlivanoglu B, Durmazlar N, Balkanci D: Computer adapted stroop colour-word conflict test as a laboratory stress model. Erciyes Med J, 2005, 27: $58-63$.

11) Fox RH, Solman A: A new technique for monitoring the deep body temperature in man from the intact skin surface. J Physiol, 1971, 212: 8-10. [Medline]

12) Karthikeyan $P$, Murugappan $M$, Yaacob S: ECG signals based mental stress assessment using wavelet transform, in 2011 IEEE International Conference on Control System Computing and Engineering (ICCSCE), 2011, 258-262.

13) Kim J: Bimodal emotion recognition using speech and physiological changes: robust speech recognition and understanding. Vienna: InTech, 2007, pp 265-280.

14) von Dawans B, Kirschbaum C, Heinrichs M: The Trier Social Stress Test for Groups (TSST-G): a new research tool for controlled simultaneous social stress exposure in a group format. Psychoneuroendocrinology, 2011, 36: 514-522. [Medline] [CrossRef]

15) Karthikeyan P, Murugappan M, Yaacob S: EMG signal based human stress level classification using wavelet packet transform, in first international conference on Intelligent Robotics, Automation and Manufacturing (IRAM), Kuala Lumpur, Malaysia, 2012.

16) Specht D: Probablistic neural networks. Neural Netw, 1990, 3: 108-118. [CrossRef] 